\title{
DEBYE MODEL FOR THE SURFACE PHONONS
}

\author{
Yu.M. Poluektov* \\ National Science Center "Kharkov Institute of Physics and Technology", \\ 1, Akademicheskaya St., 61108 Kharkov, Ukraine
}

\begin{abstract}
A quantum description of the surface waves in an isotropic elastic body without the use of the semiclassical quantization is proposed. The problem about the surface waves is formulated in the Lagrangian and Hamiltonian representations. Within the framework of the generalized Debye model, the contribution of the surface phonons ("rayleighons") to thermodynamic functions is calculated. It is emphasized that the role of the surface phonons can be significant and even decisive in lowdimensional systems, granular and porous media, and that their contribution to the total heat capacity increases with decreasing temperature.
\end{abstract}

Key words: Rayleigh surface waves, phonon, Debye model, entropy, heat capacity

PACS numbers: 63.20.-e, 63.20.Pw, 62.30.+d, 62.65.+k

\section{INTRODUCTION}

Methods of describing spatially homogeneous condensed media are fairly well developed, a large number of specific problems have been solved. Although here, too, there remain many unsolved problems associated mainly with the correct account for interparticle interactions and the description of phase transitions.

In recent years, more and more attention has been paid to both experimental and theoretical study of media wherein interphase boundaries and surfaces play an important role. It is precisely in such essentially inhomogeneous systems that there is reason to hope for the discovery of new physical effects and the production of new materials with unique properties on their basis. A simple transfer of the methods of theoretical study of homogeneous systems to the case of strongly inhomogeneous media is clearly insufficient, and therefore there is a need for the elaboration and development of special approaches. For low temperatures, the quantum-mechanical description of such systems, in particular of their electronic and phonon properties, has acquired a fundamental importance.

A widespread model of solids, which gives a correct description of thermodynamic properties in the limiting cases of low and high temperatures, is the well-known model proposed by Debye even before the creation of modern quantum theory [1]. In the Debye model for an elastic isotropic medium, it is assumed that phonons have a certain averaged velocity. However, it offers no principal difficulty to generalize the Debye model for an isotropic solid with taking into account longitudinal and transverse phonons propagating with different velocities [2]. As was shown by Rayleigh [3], in an isotropic elastic body, together with longitudinal and transverse waves, along the body surface there may propagate surface waves, the amplitude of which decreases with distance from the boundary. The quasiparticles corresponding to these waves - the surface phonons - will also give a contribution to the thermodynamic functions of a solid which is proportional to the area of its surface. This contribution can be significant and even decisive for low-dimensional samples, as well as bodies having a granular or porous structure. The questions of the theory of local and surface vibrations of a crystal lattice, as well as the literature on this issue, are considered in the book [4].

The quantization of the surface waves, that is similar to the semiclassical quantization of the photon field by Planck, was carried out by Khalatnikov in the study of heat transfer between a solid and superfluid helium [5]. However, a consistent quantum theory, as is known, was built only more than a quarter of a century after the introduction of a new world constant by Planck. At the same time, a general prescription for the transition from classical to quantum description became clear. It consists in the fact that within the framework of the classical approach there are selected generalized coordinates and canonical momenta, which, upon passing to the quantum description, are considered as operators acting in the space of wave functions and obeying the commutation relations which generalize the classical Poisson brackets. This quantization prescription is suitable both for systems of particles interacting by means of a potential $[6,7]$ and for continuous media (fields) $[2,8,9]$. For surface waves at the boundary of an isotropic solid, such a quantization method has not yet been implemented, which is the main goal of this work. On the basis of the generalized Debye model, taking into account the difference in the velocities of longitudinal and transverse sound waves [2], we calculate the contribution of the surface phonons to thermodynamic functions at arbitrary temperatures.

*Electronic address: yuripoluektov@kipt.kharkov.ua 


\section{SURFACE WAVES IN AN ISOTROPIC ELASTIC MEDIUM}

Let us first consider the classical surface wave problem. Suppose that an elastic isotropic medium fills the half-space $z<0$. Thus, the surface separating the medium and vacuum is $z=0$. The deformation vector in an elastic isotropic medium $\mathbf{u}(\mathbf{r}, z, t)$ satisfies the equation [10]

$$
\ddot{\mathbf{u}}=c_{t}^{2} \Delta \mathbf{u}+\left(c_{l}^{2}-c_{t}^{2}\right) \nabla \operatorname{div} \mathbf{u}
$$

where

$$
c_{t}=\sqrt{\frac{\mu}{\rho}}, \quad c_{l}=\sqrt{\frac{\lambda+2 \mu}{\rho}}
$$

are the velocities of the transverse and longitudinal waves, $\mu, \lambda$ are the Lame coefficients, $\rho$ is the density.

Since the system under consideration is spatially homogeneous in the $x y$-plane and inhomogeneous along the $z$-axis perpendicular to the surface, we represent the real deformation vector in the form of an expansion in two-dimensional plane waves

$$
\mathbf{u}(\mathbf{r}, z, t)=\frac{1}{\sqrt{A}} \sum_{\mathbf{k}}\left[\mathbf{u}(\mathbf{k}, z, t) e^{i \mathbf{k r}}+\mathbf{u}^{*}(\mathbf{k}, z, t) e^{-i \mathbf{k r}}\right]
$$

$A$ is the surface area. Here $\mathbf{r}$ and $\mathbf{k}$ are the two-dimensional vectors: $\mathbf{r} \equiv(x, y), \mathbf{k} \equiv\left(k_{x}, k_{y}\right)$. As is known [10], the deformation vector of a medium can be represented as the sum $\mathbf{u}=\mathbf{u}_{l}+\mathbf{u}_{t}$, where div $\mathbf{u}_{t}=0$ and rot $\mathbf{u}_{l}=0$. Each of these vectors can be expressed in terms of scalar and vector potentials

$$
\mathbf{u}_{l}=\nabla \varphi, \quad \mathbf{u}_{t}=\operatorname{rot} \psi
$$

which satisfy the wave equations

$$
\ddot{\varphi}=c_{l}^{2} \Delta \varphi, \quad \ddot{\psi}=c_{t}^{2} \Delta \psi .
$$

The potentials can be represented in a form similar to the decomposition (3):

$$
\begin{aligned}
\varphi(\mathbf{r}, z, t) & =\frac{1}{\sqrt{A}} \sum_{\mathbf{k}}\left[\varphi(\mathbf{k}, z, t) e^{i \mathbf{k r}}+\varphi^{*}(\mathbf{k}, z, t) e^{-i \mathbf{k r}}\right], \\
\boldsymbol{\psi}(\mathbf{r}, z, t) & =\frac{1}{\sqrt{A}} \sum_{\mathbf{k}}\left[\boldsymbol{\psi}(\mathbf{k}, z, t) e^{i \mathbf{k r}}+\boldsymbol{\psi}^{*}(\mathbf{k}, z, t) e^{-i \mathbf{k r}}\right] .
\end{aligned}
$$

Applying the Fourier transform in time

$$
\varphi(\mathbf{k}, z, t)=\int_{-\infty}^{\infty} \varphi(\mathbf{k}, z, \omega) e^{-i \omega t} d \omega, \quad \boldsymbol{\psi}(\mathbf{k}, z, t)=\int_{-\infty}^{\infty} \boldsymbol{\psi}(\mathbf{k}, z, \omega) e^{-i \omega t} d \omega,
$$

from the equations (4) we find the solutions bounded at $z<0$

$$
\varphi(\mathbf{k}, z, \omega)=A(\mathbf{k}, \omega) \exp \left(\gamma_{l} z\right), \quad \boldsymbol{\psi}(\mathbf{k}, z, \omega)=\mathbf{B}(\mathbf{k}, \omega) \exp \left(\gamma_{t} z\right)
$$

where

$$
\gamma_{l}=\sqrt{k^{2}-\frac{\omega^{2}}{c_{l}^{2}}}, \quad \gamma_{t}=\sqrt{k^{2}-\frac{\omega^{2}}{c_{t}^{2}}}
$$

If the radicands in (9) are positive, then the waves decay exponentially deep into the medium, and if the radicands are negative, then the solutions (8) have the form of oscillations.

The components of the vector of longitudinal deformations, with taking into account the form of the solutions (8) and the formula (4), are determined by the relations:

$$
\begin{aligned}
& u_{l x}=\frac{i}{\sqrt{A}} \sum_{\mathbf{k}} \int d \omega k_{x}\left[A(\mathbf{k}, \omega) e^{\gamma_{l} z} e^{i(\mathbf{k r}-\omega t)}-A^{*}(\mathbf{k}, \omega) e^{\gamma_{l}^{*} z} e^{-i(\mathbf{k r}-\omega t)}\right], \\
& u_{l y}=\frac{i}{\sqrt{A}} \sum_{\mathbf{k}} \int d \omega k_{y}\left[A(\mathbf{k}, \omega) e^{\gamma_{l} z} e^{i(\mathbf{k r}-\omega t)}-A^{*}(\mathbf{k}, \omega) e^{\gamma_{l}^{*} z} e^{-i(\mathbf{k r}-\omega t)}\right], \\
& u_{l z}=\frac{1}{\sqrt{A}} \sum_{\mathbf{k}} \int d \omega\left[\gamma_{l} A(\mathbf{k}, \omega) e^{\gamma_{l} z} e^{i(\mathbf{k r}-\omega t)}+\gamma_{l}^{*} A^{*}(\mathbf{k}, \omega) e^{\gamma_{l}^{*} z} e^{-i(\mathbf{k r}-\omega t)}\right] .
\end{aligned}
$$


The components of the vector of transverse deformations, with account of (8) and (4), are given by the formulas:

$$
\begin{aligned}
& u_{t x}=\frac{1}{\sqrt{A}} \sum_{\mathbf{k}} \int d \omega\left\{\left[i k_{y} B_{z}(\mathbf{k}, \omega)-\gamma_{t} B_{y}(\mathbf{k}, \omega)\right] e^{\gamma_{t} z} e^{i(\mathbf{k r}-\omega t)}+\left[-i k_{y} B_{z}^{*}(\mathbf{k}, \omega)-\gamma_{t}^{*} B_{y}^{*}(\mathbf{k}, \omega)\right] e^{\gamma_{t}^{*} z} e^{-i(\mathbf{k r}-\omega t)}\right\}, \\
& u_{t y}=\frac{1}{\sqrt{A}} \sum_{\mathbf{k}} \int d \omega\left\{\left[-i k_{x} B_{z}(\mathbf{k}, \omega)+\gamma_{t} B_{x}(\mathbf{k}, \omega)\right] e^{\gamma_{t} z} e^{i(\mathbf{k r}-\omega t)}+\left[i k_{x} B_{z}^{*}(\mathbf{k}, \omega)+\gamma_{t}^{*} B_{x}^{*}(\mathbf{k}, \omega)\right] e^{\gamma_{t}^{*} z} e^{-i(\mathbf{k r}-\omega t)}\right\}, \\
& u_{t z}=\frac{i}{\sqrt{A}} \sum_{\mathbf{k}} \int d \omega\left\{\left[k_{x} B_{y}(\mathbf{k}, \omega)-k_{y} B_{x}(\mathbf{k}, \omega)\right] e^{\gamma_{t} z} e^{i(\mathbf{k r}-\omega t)}-\left[k_{x} B_{y}^{*}(\mathbf{k}, \omega)-k_{y} B_{x}^{*}(\mathbf{k}, \omega)\right] e^{\gamma_{t}^{*} z} e^{-i(\mathbf{k r}-\omega t)}\right\} .
\end{aligned}
$$

The formulas (10), (11) give a general solution for the deformation vector under arbitrary boundary conditions, which is bounded in the half-space $z<0$.

Let us obtain a particular solution, provided that no external forces act on the surface. The external force acting per unit surface area is determined by the expression $f_{i}=\sigma_{i k} n_{k}$ [10], where $\mathbf{n}$ is the outward normal vector to the surface, which in the case under consideration is directed along the $z$-axis, so that $\mathbf{n}=(0,0,1)$. Assuming that at $z=0$ the force is equal to zero, we have the boundary conditions $\sigma_{x z}=\sigma_{y z}=\sigma_{z z}=0$, which, with taking into account the form of the stress tensor for an isotropic elastic medium, have the form

$$
\frac{\partial u_{x}}{\partial z}+\frac{\partial u_{z}}{\partial x}=0, \quad \frac{\partial u_{y}}{\partial z}+\frac{\partial u_{z}}{\partial y}=0, \quad(1-\sigma) \frac{\partial u_{z}}{\partial z}+\sigma\left(\frac{\partial u_{x}}{\partial x}+\frac{\partial u_{y}}{\partial y}\right)=0
$$

where $\sigma$ is the Poisson coefficient. Using the formulas (10), (11), by means of the boundary conditions (12) we obtain the algebraic equations for the expansion coefficients in these formulas

$$
\begin{aligned}
& 2 i k_{x} \gamma_{l} A+k_{x} k_{y} B_{x}-\left(\gamma_{t}^{2}+k_{x}^{2}\right) B_{y}+i k_{y} \gamma_{t} B_{z}=0 \\
& 2 i k_{y} \gamma_{l} A+\left(\gamma_{t}^{2}+k_{y}^{2}\right) B_{x}-k_{x} k_{y} B_{y}-i k_{x} \gamma_{t} B_{z}=0 \\
& {\left[(1-\sigma) \gamma_{l}^{2}-\sigma\left(k_{x}^{2}+k_{y}^{2}\right)\right] A+i(1-2 \sigma) \gamma_{t}\left(k_{x} B_{y}-k_{y} B_{x}\right)=0}
\end{aligned}
$$

To shorten the notation we used the designations $A(\mathbf{k}, \omega) \equiv A, \mathbf{B}(\mathbf{k}, \omega) \equiv \mathbf{B}$. It is also convenient to introduce the designations

$$
B_{+}=k^{-1}\left(k_{x} B_{x}+k_{y} B_{y}\right), \quad B_{-}=k^{-1}\left(k_{x} B_{y}-k_{y} B_{x}\right),
$$

where $k^{2}=k_{x}^{2}+k_{y}^{2}$. Then the system of equations (13) takes the form

$$
\begin{gathered}
i B_{z}=\alpha_{t} B_{+}, \\
2 i \alpha_{l} A-\left(1+\alpha_{t}^{2}\right) B_{-}=0 \\
(2-y) A+2 i \alpha_{t} B_{-}=0
\end{gathered}
$$

wherein

$$
\begin{aligned}
& y \equiv \frac{\omega^{2}}{c_{t}^{2} k^{2}}, \quad \gamma_{l}=\alpha_{l} k, \quad \gamma_{t}=\alpha_{t} k \\
& \alpha_{l} \equiv \sqrt{1-\xi^{2} y}, \quad \alpha_{t} \equiv \sqrt{1-y}, \quad \xi^{2}=\left(\frac{c_{t}}{c_{l}}\right)^{2}=\frac{(1 / 2-\sigma)}{(1-\sigma)}
\end{aligned}
$$

The compatibility condition for a system of linear homogeneous equations gives the equation

$$
4 \alpha_{t} \alpha_{l}=(2-y)^{2}
$$

which, with account of the formulas (17), is reduced to the well-known cubic equation [10]

$$
y^{3}-8 y^{2}+8\left(3-2 \xi^{2}\right) y-16\left(1-\xi^{2}\right)=0
$$

determining the dispersion law of surface waves. The parameter $y$ is a real number less than one, the value of which is determined by the ratio of the transverse and longitudinal wave velocities. Thus, the velocity of surface waves

$$
c_{s}=\sqrt{y} c_{t}
$$


is less than the velocity of body waves. The radicands in (17) are positive for all values of $y$ and the quantities $\alpha_{l}, \alpha_{t}$ are real, while $\alpha_{l}>\alpha_{t}$. In the case when there is a relationship $\omega=\omega_{0}(\mathbf{k})$ between the frequency and the wavenumber, the amplitudes in the formulas (10), (11) should be taken in the form

$$
A(\mathbf{k}, \omega)=\tilde{A}(\mathbf{k}) \delta\left(\omega-\omega_{0}(\mathbf{k})\right), \quad \mathbf{B}(\mathbf{k}, \omega)=\tilde{\mathbf{B}}(\mathbf{k}) \delta\left(\omega-\omega_{0}(\mathbf{k})\right),
$$

where $\tilde{A}(\mathbf{k}) \equiv A\left(\mathbf{k}, \omega_{0}(\mathbf{k})\right), \tilde{\mathbf{B}}(\mathbf{k}) \equiv \mathbf{B}\left(\mathbf{k}, \omega_{0}(\mathbf{k})\right)$. In our case $\omega_{0}(\mathbf{k})=\sqrt{y} c_{t} k$. In the following we will omit the tilde sign. The relations (13)-(16) make it possible to express the deformation vector only in terms of one amplitude $A(\mathbf{k})$ :

$$
\begin{aligned}
& u_{x}(\mathbf{r}, z, t)=\frac{i}{\sqrt{A}} \sum_{\mathbf{k}}\left\{k_{x} g_{1}(z, k)\left[A(\mathbf{k}) e^{i\left(\mathbf{k r}-\omega_{0}(\mathbf{k}) t\right)}-A^{*}(\mathbf{k}) e^{-i\left(\mathbf{k r}-\omega_{0}(\mathbf{k}) t\right)}\right]\right\}, \\
& u_{y}(\mathbf{r}, z, t)=\frac{i}{\sqrt{A}} \sum_{\mathbf{k}}\left\{k_{y} g_{1}(z, k)\left[A(\mathbf{k}) e^{i\left(\mathbf{k r}-\omega_{0}(\mathbf{k}) t\right)}-A^{*}(\mathbf{k}) e^{-i\left(\mathbf{k r}-\omega_{0}(\mathbf{k}) t\right)}\right]\right\}, \\
& u_{z}(\mathbf{r}, z, t)=\frac{\alpha_{l}}{\sqrt{A}} \sum_{\mathbf{k}}\left\{k g_{2}(z, k)\left[A(\mathbf{k}) e^{i\left(\mathbf{k r}-\omega_{0}(\mathbf{k}) t\right)}+A^{*}(\mathbf{k}) e^{-i\left(\mathbf{k r}-\omega_{0}(\mathbf{k}) t\right)}\right]\right\} .
\end{aligned}
$$

Here, the following functions are defined

$$
g_{1}(z, k) \equiv e^{\alpha_{l} k z}-\frac{2 \alpha_{l} \alpha_{t}}{1+\alpha_{t}^{2}} e^{\alpha_{t} k z}, \quad g_{2}(z, k) \equiv e^{\alpha_{l} k z}-\frac{2}{1+\alpha_{t}^{2}} e^{\alpha_{t} k z}
$$

After defining the time-dependent amplitudes

$$
A(\mathbf{k}, t)=A(\mathbf{k}) e^{-i \omega_{0}(\mathbf{k}) t}, \quad A^{*}(\mathbf{k}, t)=A^{*}(\mathbf{k}) e^{i \omega_{0}(\mathbf{k}) t},
$$

and introducing the new amplitudes

$$
X(\mathbf{k}, t)=X^{*}(-\mathbf{k}, t) \equiv A(\mathbf{k}, t)+A^{*}(-\mathbf{k}, t),
$$

the components of the deformation vector can be written as

$$
\begin{aligned}
& u_{x}(\mathbf{r}, z, t)=\frac{i}{\sqrt{A}} \sum_{\mathbf{k}} k_{x} g_{1}(z, k) X(\mathbf{k}, t) e^{i \mathbf{k r}}, \\
& u_{y}(\mathbf{r}, z, t)=\frac{i}{\sqrt{A}} \sum_{\mathbf{k}} k_{y} g_{1}(z, k) X(\mathbf{k}, t) e^{i \mathbf{k r}}, \\
& u_{z}(\mathbf{r}, z, t)=\frac{\alpha_{l}}{\sqrt{A}} \sum_{\mathbf{k}} k g_{2}(z, k) X(\mathbf{k}, t) e^{i \mathbf{k r}}
\end{aligned}
$$

Before proceeding to quantizing surface excitations, we present the Lagrangian and the Hamiltonian formulations of the problem.

\section{LAGRANGIAN AND HAMILTONIAN FORMULATIONS OF THE SURFACE WAVES PROBLEM}

The density of the Lagrangian function, which leads to the equations for waves in an isotropic elastic medium (1), has the form

$$
\Lambda(\mathbf{r}, z, t)=\frac{\rho}{2} \dot{\mathbf{u}}^{2}-\frac{\lambda}{2}(\operatorname{div} \mathbf{u})^{2}-\frac{\mu}{2}\left(\nabla_{j} u_{i} \nabla_{i} u_{j}+\nabla_{j} u_{i} \nabla_{j} u_{i}\right),
$$

and to find the complete Lagrangian function one should integrate (27) over the entire volume occupied by the medium

$$
L=\int_{A} d \mathbf{r} \int_{-\infty}^{0} d z \Lambda(\mathbf{r}, z)
$$

Using the obtained expressions for the components of the deformation vector (26), for which the boundary conditions (12) are fulfilled, and integrating over spatial coordinates, we obtain the Lagrangian function in the form

$$
L=\frac{\rho \alpha_{l}}{4} \Theta(y) \sum_{\mathbf{k}}\left[k|\dot{X}(\mathbf{k})|^{2}-c_{s}^{2} k^{3}|X(\mathbf{k})|^{2}\right],
$$

where

$$
\Theta(y) \equiv \frac{\left[16(1-y)^{2}+(2-y)^{4}\right]}{(1-y)(2-y)^{4}}-2 \frac{(2+y)}{(2-y)}=\frac{y^{2}\left(8-16 y+11 y^{2}-2 y^{3}\right)}{(1-y)(2-y)^{4}}
$$


Instead of the complex quantities in (29), one should pass to the real quantities $X(\mathbf{k})=X^{\prime}(\mathbf{k})+i X^{\prime \prime}(\mathbf{k})$ and consider $X^{\prime}(\mathbf{k}), X^{\prime \prime}(\mathbf{k})$ as generalized coordinates and $\dot{X}^{\prime}(\mathbf{k}), \dot{X}^{\prime \prime}(\mathbf{k})$ as generalized velocities, on which the Lagrangian function depends. However, it should be taken into account that the Lagrangian must be expressed in terms of independent coordinates and velocities. Meanwhile, due to the condition (25), the coordinates at the oppositely directed wave vectors $\mathbf{k}$ and $\mathbf{- k}$ are linked by the relations

$$
X^{\prime}(\mathbf{k}, t)=X^{\prime}(-\mathbf{k}, t), \quad X^{\prime \prime}(\mathbf{k}, t)=-X^{\prime \prime}(-\mathbf{k}, t)
$$

and therefore are not independent. To pass to independent variables in the Lagrangian (29), we decompose it into two terms, each of which contains a summation over all wave vectors except for the oppositely directed ones. Due to the conditions (31), the contribution to the Lagrangian of each such term will be the same. Therefore, the Lagrangian (29) should be multiplied by two, and the summation should be carried out only over those wave vectors among which there are no the oppositely directed ones. For example, we may sum over all wave vectors having $k_{z}>0$. As a result, the Lagrangian, expressed in terms of the real independent even and odd (31) coordinates and velocities, takes the form

$$
L=\frac{\rho \alpha_{l} \Theta(y)}{2} \sum_{\mathbf{k}}\left[k \dot{X}^{\prime 2}(\mathbf{k})-c_{s}^{2} k^{3} X^{\prime 2}(\mathbf{k})\right]+\frac{\rho \alpha_{l} \Theta(y)}{2} \sum_{\mathbf{k}}\left[k \dot{X}^{\prime \prime 2}(\mathbf{k})-c_{s}^{2} k^{3} X^{\prime \prime 2}(\mathbf{k})\right] .
$$

Here, the symbol $\frown$ above the sum sign, as explained above, means a summation over vectors among which there are no the oppositely directed ones. The Euler-Lagrange equations give rise to the equations of motion

$$
\ddot{X}^{\prime}(\mathbf{k})+c_{s}^{2} k^{2} X^{\prime}(\mathbf{k})=0, \quad \ddot{X}^{\prime \prime}(\mathbf{k})+c_{s}^{2} k^{2} X^{\prime \prime}(\mathbf{k})=0 .
$$

Let us move on to the Hamiltonian description, defining the canonical momenta

$$
\Pi^{\prime}(\mathbf{k})=\frac{\partial L}{\partial \dot{X}^{\prime}(\mathbf{k})}=\rho \alpha_{l} \Theta(y) k \dot{X}^{\prime}(\mathbf{k}), \quad \Pi^{\prime \prime}(\mathbf{k})=\frac{\partial L}{\partial \dot{X}^{\prime \prime}(\mathbf{k})}=\rho \alpha_{l} \Theta(y) k \dot{X}^{\prime \prime}(\mathbf{k}) .
$$

We introduce the Hamilton function

$$
H=\sum_{\mathbf{k}}\left(\dot{X}^{\prime}(\mathbf{k}) \frac{\partial L}{\partial \dot{X}^{\prime}(\mathbf{k})}+\dot{X}^{\prime \prime}(\mathbf{k}) \frac{\partial L}{\partial \dot{X}^{\prime \prime}(\mathbf{k})}\right)-L=H^{\prime}+H^{\prime \prime}
$$

where $H^{\prime}, H^{\prime \prime}$ are the Hamiltonians expressed in terms of the coordinates and momenta with one and two primes:

$$
\begin{aligned}
& H^{\prime}=\frac{\rho \alpha_{l} \Theta(y)}{2} \sum_{\mathbf{k}}\left[\frac{\Pi^{\prime 2}(\mathbf{k})}{\rho^{2} \alpha_{l}^{2} \Theta^{2}(y)}+c_{s}^{2} k^{3} X^{\prime 2}(\mathbf{k})\right] \\
& H^{\prime \prime}=\frac{\rho \alpha_{l} \Theta(y)}{2} \sum_{\mathbf{k}}^{\frown}\left[\frac{\Pi^{\prime \prime 2}(\mathbf{k})}{\rho^{2} \alpha_{l}^{2} \Theta^{2}(y)}+c_{s}^{2} k^{3} X^{\prime \prime 2}(\mathbf{k})\right] .
\end{aligned}
$$

Since these Hamiltonians have the same form, it suffices to consider only the Hamiltonian expressed in terms of the coordinates and momenta with one prime. From the Hamilton equations

$$
\dot{\Pi}^{\prime}(\mathbf{k})=-\frac{\partial H^{\prime}}{\partial X^{\prime}(\mathbf{k})}, \quad \dot{X}^{\prime}(\mathbf{k})=\frac{\partial H^{\prime}}{\partial \Pi^{\prime}(\mathbf{k})}
$$

there follow the equations of motion

$$
\dot{\Pi}^{\prime}(\mathbf{k})=-\rho \alpha_{l} \Theta(y) c_{s}^{2} k^{3} X^{\prime}(\mathbf{k}), \quad \dot{X}^{\prime}(\mathbf{k})=\frac{\Pi^{\prime}(\mathbf{k})}{\rho \alpha_{l} \Theta(y) k} .
$$

These equations with account of the definition of momenta (34), of course, can also be written in the form of the second-order equations for an oscillator (33). 


\section{QUANTIZATION OF SURFACE WAVES}

Now we pass from the classical to the consistently quantum description of surface excitations without the use of the semiclassical approximation [5]. In this case, the coordinates and momenta should be considered as operators obeying the following well-known commutation relations:

$$
\begin{aligned}
& {\left[X^{\prime}(\mathbf{k}), \Pi^{\prime}\left(\mathbf{k}^{\prime}\right)\right] \equiv X^{\prime}(\mathbf{k}) \Pi^{\prime}\left(\mathbf{k}^{\prime}\right)-\Pi^{\prime}\left(\mathbf{k}^{\prime}\right) X^{\prime}(\mathbf{k})=i \hbar \Delta\left(\mathbf{k}-\mathbf{k}^{\prime}\right),} \\
& {\left[X^{\prime}(\mathbf{k}), X^{\prime}\left(\mathbf{k}^{\prime}\right)\right]=\left[\Pi^{\prime}(\mathbf{k}), \Pi^{\prime}\left(\mathbf{k}^{\prime}\right)\right]=0 .}
\end{aligned}
$$

The same commutation relations hold for the coordinates and momenta with two primes, and all variables with one prime commute with all variables with two primes. It is convenient to pass from the Hermitian operators $X^{\prime}(\mathbf{k})=X^{\prime+}(\mathbf{k})$ and $\Pi^{\prime}(\mathbf{k})=\Pi^{\prime+}(\mathbf{k})$ to the new non-Hermitian operators $a^{+}(\mathbf{k}), a(\mathbf{k})$, which, as we will see, have the meaning of the operators of creation and annihilation of surface phonons

$$
\begin{aligned}
& X^{\prime}(\mathbf{k})=C(\mathbf{k}) a(\mathbf{k})+C^{*}(\mathbf{k}) a^{+}(\mathbf{k}), \\
& \Pi^{\prime}(\mathbf{k})=B(\mathbf{k}) a(\mathbf{k})+B^{*}(\mathbf{k}) a^{+}(\mathbf{k}),
\end{aligned}
$$

where the coefficients $C(\mathbf{k}), B(\mathbf{k})$ are $c$-numbers. The commutation relations (39) will be satisfied if the following well-known commutation relations are required to hold

$$
\begin{aligned}
& {\left[a(\mathbf{k}), a^{+}\left(\mathbf{k}^{\prime}\right)\right] \equiv a(\mathbf{k}) a^{+}\left(\mathbf{k}^{\prime}\right)-a^{+}\left(\mathbf{k}^{\prime}\right) a(\mathbf{k})=\Delta\left(\mathbf{k}-\mathbf{k}^{\prime}\right),} \\
& {\left[a(\mathbf{k}), a\left(\mathbf{k}^{\prime}\right)\right]=\left[a^{+}(\mathbf{k}), a^{+}\left(\mathbf{k}^{\prime}\right)\right]=0 .}
\end{aligned}
$$

In addition, in order for the transformation (40) to be canonical, the following condition should hold

$$
C(\mathbf{k}) B^{*}(\mathbf{k})-C^{*}(\mathbf{k}) B(\mathbf{k})=i \hbar .
$$

In the Hamilton operators (36), which we write in the form (similarly for $H^{\prime \prime}$ )

$$
H^{\prime}=\sum_{\mathbf{k}}\left[\psi(k) \Pi^{\prime 2}(\mathbf{k})+\varphi(k) X^{\prime 2}(\mathbf{k})\right],
$$

where for brevity the designations $\psi(k) \equiv 1 / 2 \rho \alpha_{l} \Theta(y) k, \varphi(k) \equiv \rho \alpha_{l} \Theta(y) c_{s}^{2} k^{3} / 2$ are used, it is also necessary to pass to the new operators $a^{+}(\mathbf{k}), a(\mathbf{k})$. When substituting the relations (40) into (43) we require that only the operators of the form $a(\mathbf{k}) a^{+}(\mathbf{k})$ or $a^{+}(\mathbf{k}) a(\mathbf{k})$ remain in the resulting Hamiltonian, while the operators $a^{2}(\mathbf{k})$ and $a^{+2}(\mathbf{k})$ drop out. This entails the fulfillment of the condition

$$
\psi(k) B^{2}(\mathbf{k})+\varphi(k) C^{2}(\mathbf{k})=0 .
$$

The condition (44) will be satisfied if

$$
B^{2}(k)=2 / \hbar \rho \alpha_{l} \Theta(y) c_{s} k^{2}, \quad C^{2}(k)=-\hbar / 2 \rho \alpha_{l} \Theta(y) c_{s} k^{2} .
$$

When extracting the root the signs can be chosen arbitrarily, since this will not affect the final result. Thus, the canonical transformation (40) takes the form

$$
\begin{aligned}
& X^{\prime}(\mathbf{k})=i \frac{\hbar}{k \sqrt{\hbar \rho \alpha_{l} 2 \Theta(y) c_{s}}}\left[a(\mathbf{k})-a^{+}(\mathbf{k})\right], \\
& \Pi^{\prime}(\mathbf{k})=k \sqrt{\frac{\hbar \rho \alpha_{l} \Theta(y) c_{s}}{2}}\left[a(\mathbf{k})+a^{+}(\mathbf{k})\right] .
\end{aligned}
$$

As a result, using the commutation relations (41), we find

$$
H^{\prime}=\sum_{\mathbf{k}} \hbar c_{s} k\left[a^{+}(\mathbf{k}) a(\mathbf{k})+\frac{1}{2}\right] .
$$

The diagonalization of the Hamiltonian $H^{\prime \prime}$ can be carried out in a similar way. When calculating the thermodynamic quantities, the Hamiltonians $H^{\prime}$ and $H^{\prime \prime}$ make the same contribution, so that one can use the doubled operator (47) and the total Hamiltonian takes the form

$$
H=2 \sum_{\mathbf{k}} \hbar c_{s} k\left[a^{+}(\mathbf{k}) a(\mathbf{k})+\frac{1}{2}\right] .
$$


The Hamiltonian (48) differs from the Hamiltonian of bulk phonons in the value of the velocity and in that the summation is carried out over two-dimensional wave vectors. The surface two-dimensional phonons, having velocity $c_{s}$, can naturally be called "rayleighons". Note that the quanta of vibrations of the surface of liquid helium are called riplons $[11,12]$.

\section{CONTRIBUTION OF "RAYLEIGHONS" TO THERMODYNAMIC FUNCTIONS}

The average values of the surface thermodynamic functions are calculated by means of the statistical operator

$$
\rho=\exp \beta(F-H)
$$

where the Hamiltonian is defined by the formula (48), and $\beta=1 / T$ is the inverse temperature. From the normalization condition $\operatorname{Sp} \rho=1$ we find the surface free energy

$$
F=\frac{\hbar}{2} \sum_{\mathbf{k}} c_{s} k+T \sum_{\mathbf{k}} \ln \left(1-e^{-\beta \hbar c_{s} k}\right) .
$$

Here the summation, as noted above, is carried out over the two-dimensional wave vectors $\mathbf{k} \equiv\left(k_{x}, k_{y}\right)$. The first term in (50) determines the contribution of zero oscillations. In (50) we pass from summation to integration in which the upper limit of integration over the magnitude of the wave vector is determined, as in the bulk case, by the Debye relation $k_{D}=\left(6 \pi^{2} n\right)^{1 / 3}, n=N / V$ is the particle number density. As a result, we obtain the surface free energy in the form

$$
F=\frac{A k_{D}^{2}}{12 \pi}\left\{\Theta_{s}+\frac{3}{2} T\left[2 \ln \left(1-e^{-\tau^{-1}}\right)-D_{2}\left(\tau^{-1}\right)\right]\right\},
$$

where $\Theta_{s} \equiv \hbar c_{s} k_{D}$ is the "surface" Debye energy, $\tau \equiv T / \Theta_{s}$, and the Debye functions are defined by the formula

$$
D_{n}(x)=\frac{n}{x^{n}} \int_{0}^{x} \frac{z^{n} d z}{e^{z}-1} \quad(n \geq 1) .
$$

The surface entropy $S=-(\partial F / \partial T)_{V A}$ has the form

$$
S=\frac{A k_{D}^{2}}{8 \pi}\left[3 D_{2}\left(\tau^{-1}\right)-2 \ln \left(1-e^{-\tau^{-1}}\right)\right] .
$$

From here we find the heat capacity at a constant volume and surface area

$$
C_{V A}=T\left(\frac{\partial S}{\partial T}\right)_{V A}=\frac{A k_{D}^{2}}{4 \pi \tau}\left[3 \tau D_{2}\left(\tau^{-1}\right)-\frac{2}{e^{\tau^{-1}}-1}\right] .
$$

Let us consider the behavior of the surface heat capacity (54) in the limit of low and high temperatures. Since $D_{2}(x) \approx 4 x^{-2} \zeta(3)$ at $x \ll 1$, we find at $\tau \ll 1$ :

$$
C_{V A} \approx \frac{3 \zeta(3)}{\pi} A k_{D}^{2}\left(\frac{T}{\Theta_{s}}\right)^{2}=\frac{3 \zeta(3)}{\pi \hbar^{2} c_{s}^{2}} A T^{2},
$$

$\zeta(s)$ is the Riemann zeta function. Note that the structure of this formula is similar to the structure of the corresponding formula for the bulk case of the usual Debye theory

$$
C_{V} \approx \frac{2 \pi^{2}}{5} V k_{D}^{3}\left(\frac{T}{\Theta_{D}}\right)^{3}=\frac{2 \pi^{2}}{5 \hbar^{3} c_{D}^{3}} V T^{3}
$$

where $c_{D}$ is the average phonon velocity according to Debye $[13,14]$. In the two-parameter theory [2], the formula for the low-temperature bulk heat capacity takes the form

$$
C_{V} \approx \frac{2 \pi^{2}}{5} V k_{D}^{3} \cdot f(\chi)\left(\frac{T}{\Theta}\right)^{3}=\frac{2 \pi^{2}}{5 \hbar^{3}}\left(\frac{3}{2 c_{t}^{2}+c_{l}^{2}}\right)^{3 / 2} V f(\chi) T^{3}
$$


where $\Theta^{2}=\frac{1}{3}\left(2 \Theta_{t}^{2}+\Theta_{l}^{2}\right), \operatorname{tg} \chi=\sqrt{2} \frac{\Theta_{t}}{\Theta_{l}}, \Theta_{t}=\hbar c_{t} k_{D}, \Theta_{l}=\hbar c_{l} k_{D}, f(\chi) \equiv \frac{1}{3^{5 / 2}}\left(\frac{1}{\cos ^{3} \chi}+\frac{2^{5 / 2}}{\sin ^{3} \chi}\right)$.

The calculation of the surface contribution to the phonon heat capacity has received a considerable attention. The quadratic dependence on temperature was obtained in [15], and subsequently the form of the coefficient before $T^{2}$ was refined in other works [4]. In [16-18], there was obtained a formula for the surface heat capacity in various approaches, which in the notation of $(17),(19),(20)$ can be represented in the form $[6,16]$

$$
\bar{C}_{A}=\frac{3 \zeta(3)}{\pi \hbar^{2} c_{s}^{2}} \cdot y \frac{\left(2 \xi^{4}-3 \xi^{2}+3\right)}{4\left(1-\xi^{2}\right)} A T^{2} .
$$

In the formulas (55), (58) the coefficients at $T^{2}$ differ in value insignificantly. So, for the Poisson coefficient $\sigma=1 / 2$ and $\xi^{2}=0$ we have $\bar{C}_{A} / C_{V A} \approx 0.685$, and for $\sigma=0$ and $\xi^{2}=1 / 2$ the ratio $\bar{C}_{A} / C_{V A} \approx 0.763$. The calculation of the surface heat capacity according to the formula (55) gives a slightly higher value of the heat capacity than the calculation according to the formula (58). Despite the small quantitative difference, the question about the reasons for the difference between the early formula (58) and the seemingly more natural formula (55) requires an additional consideration.

In the case of high temperatures $\tau>1$ the surface heat capacity, like the bulk heat capacity, tends to a constant value:

$$
C_{V A} \approx \frac{A k_{D}^{2}}{4 \pi}\left[1-\frac{1}{24}\left(\frac{\Theta_{s}}{T}\right)^{2}\right] .
$$

Let there be a sample in the shape of a cube with the edge length $L$, volume $V=L^{3}$ and surface area $A \approx L^{2}$. Let us estimate the ratio of the surface and bulk heat capacities in such a sample. At high temperatures, when $C_{V}=3 N$, we have

$$
\frac{C_{V A}}{C_{V}} \approx \frac{A k_{D}^{2}}{4 \pi} \frac{1}{3 N}=a \frac{l}{L}
$$

where $l=n^{-1 / 3}$ is the average distance between atoms, $a=\left(6 \pi^{2}\right)^{1 / 3} / 12 \pi \approx 0.4$. Thus, at high temperatures for macroscopic samples $L \gg l$ the surface heat capacity is small in comparison with the bulk heat capacity.

Let us consider the case of low temperatures, when for the bulk heat capacity the relation $C_{V} \approx \frac{12 \pi^{4}}{5} N\left(\frac{T}{\Theta}\right)^{3}$ holds. In this case

$$
\frac{C_{V A}}{C_{V}} \approx \frac{15 \zeta(3)}{\pi^{4}} \frac{A k_{D}^{2}}{12 \pi N}\left(\frac{\Theta}{\Theta_{s}}\right)^{2} \frac{\Theta}{T}
$$

With decreasing temperature this ratio increases, and at a certain temperature $T_{*}$ the surface and bulk heat capacities become equal. From (61) it follows that

$$
\frac{T_{*}}{\Theta}=\frac{15 \zeta(3)}{\pi^{4}} a \frac{l}{L}\left(\frac{\Theta}{\Theta_{s}}\right)^{2} \approx 0.075 \frac{l}{L},
$$

where it is assumed that $\Theta \approx \Theta_{s}$. For samples with sizes of the order of a nanometer $L \approx 10^{-7} \mathrm{~cm}$, and, taking into account that $l \approx 10^{-8} \mathrm{~cm}$, the ratio $T_{*} / \Theta \approx 0.01$. Since the Debye energy has the order of magnitude $\Theta \approx 10^{2} K$, then the law for the heat capacity $C_{V A} \approx T^{2}$ in samples of such size can be observed at helium temperatures. The contribution of the surface heat capacity can also be the main one in porous and granular materials.

In the bulk case the low-temperature heat capacity, according to the Debye theory [1], is proportional to the cube of temperature, and in the two-dimensional case, when the contribution of the surface phonons is taken into account, it is proportional to the square of temperature. This indicates that the temperature exponent in the low-temperature behavior of the heat capacity coincides with the dimension of space. In this regard, it is interesting to calculate the entropy and heat capacity of "one-dimensional" phonons. Such a situation with the quasi-one-dimensional phonons could be realized in long cylindrical filaments of small radius. The propagation of the classical surface waves under conditions of cylindrical geometry was studied in many works [19]. For a qualitative study of a one-dimensional system of phonons, it is sufficient to assume that in the expression for the free energy (50) the summation is performed over wave vectors oriented in one direction. In this case, we find

$$
F=\frac{L k_{D}}{4 \pi}\left\{\Theta_{1}+4 T\left[\ln \left(1-e^{-\tau^{-1}}\right)-D_{1}\left(\tau^{-1}\right)\right]\right\},
$$


where $L$ is the length of a sample, $\Theta_{1}=\hbar c_{1} k_{D}, c_{1}$ is the velocity of the "one-dimensional" phonons, and as before $k_{D}=\left(6 \pi^{2} n\right)^{1 / 3}$. From (63) there follow the expressions for the "one-dimensional" entropy and heat capacity:

$$
\begin{gathered}
S=\frac{L k_{D}}{\pi}\left[2 D_{1}\left(\tau^{-1}\right)-\ln \left(1-e^{-\tau^{-1}}\right)\right] \\
C_{V L}=T\left(\frac{\partial S}{\partial T}\right)_{V L}=\frac{L k_{D}}{\pi \tau}\left[2 \tau D_{1}\left(\tau^{-1}\right)-\frac{1}{e^{\tau^{-1}}-1}\right] .
\end{gathered}
$$

In the low-temperature limit $\tau \ll 1$, taking into account that $D_{1}(x) \approx \pi^{2} / 6 x$, from here we get:

$$
S=C_{V L} \approx \frac{\pi L k_{D}}{3}\left(\frac{T}{\Theta_{1}}\right)
$$

As could be expected, the low-temperature entropy and heat capacity of a system of "one-dimensional" phonons are proportional to temperature.

\section{CONCLUSION}

A method for quantizing surface elastic waves in an isotropic solid without the use of the semiclassical approximation is proposed, and in the Debye approach the contribution of the surface phonons ("rayleighons") to thermodynamic functions is calculated. In agreement with the previous works [15-18] it is shown that in the limit of low temperatures the contribution of the surface phonons is proportional to the square of temperature, while the value of the proportionality coefficient is somewhat different from the earlier results [15-18]. It is also shown that in the one-dimensional case at low temperatures the dependence of the heat capacity is linear. Thus, the exponent in the temperature dependence of the phonon heat capacity in the low-temperature limit is determined by the spatial dimensionality of a system.

There are two extreme points of view on the Debye model. Often this model is given an unduly fundamental meaning and, when processing experimental data, observable quantities are adjusted to the relations of theory assuming that the Debye energy depends on temperature. The opposite point of view is that the relations of the Debye theory are considered as rough interpolation formulas [13,14]. The Debye model, of course, is an approximate and rather simple (which is its value) model of the solid body, but there is reason to assert that its value is not limited only to the possibility of constructing a single interpolation formula that would correctly describe the behavior of the solid body in the limit of low and high temperatures. This model allows further development and generalization, for example, accounting for the difference in the velocities of longitudinal and transverse phonons [2], the interaction of phonons $[8,9]$, and, as shown in this article, it can be extended to describe surface phenomena in solids.

When analyzing thermodynamic and kinetic properties of crystals whose anisotropy is not large and the considered effects are not associated with the existence of singled-out directions in crystals, it is possible to use with a good accuracy a more simple model of an isotropic medium after choosing its parameters in an optimal way [20]. It was shown in [2] that the previously proposed method of describing the elastic properties of crystals on the basis of a comparison with an isotropic medium [20] follows from the requirement of the maximal closeness of the free energies of a crystal and an isotropic medium. The two-parameter Debye model for an isotropic medium with effective elastic moduli [2] can be a good approximation for describing the properties of crystals. All the general remarks made above refer, in particular, to the further development of the considered in this article Debye model for the surface phonons.

The author is grateful to A.S. Kovalev for helpful comments. 
[1] P. Debye, Ann. Phys. 39(4), 789 (1912). doi:10.1002/andp.19123441404

[2] Yu.M. Poluektov, East Eur. J. Phys. 5, №3, 4 (2018); arXiv:2004.06658v1 [cond-mat.stat-mech].

[3] J. Rayleigh, Proc. London Math. Soc. s1-17(1), 4 (1885). doi:10.1112/plms/s1-17.1.4

[4] A. Maradudin, Defects and vibrational spectrum of crystals, Mir, Moscow, 432 p. (1968).

[5] I.M. Khalatnikov, Theory of superfluidity, Nauka, Moscow, 320 p. (1971).

[6] A. Maradudin, E. Montroll, G. Weiss, Theory of lattice dynamics in the harmonic approximation, Acad. Press, 319 p. (1968).

[7] A.M. Kosevich, Foundation of crystal lattice mechanics, Nauka, Moscow, 280 p. (1972).

[8] Yu.M. Poluektov, Low Temp. Phys. 41, 922 (2015). doi:10.1063/1.4936228

[9] Yu.M. Poluektov, East Eur. J. Phys. 3, №3, 35 (2016).

[10] L.D. Landau, E.M. Lifshitz, Theory of elasticity, Vol. 7, Butterworth-Heinemann (3rd ed.), 196 p. (1986).

[11] V.B. Shikin, Y.P. Monarkha, Two-dimensional charged systems in helium, Nauka, Moscow, 156 p. (1989).

[12] Y. Monarkha, K. Kono, Two-dimensional Coulomb liquids and solids, Springer-Verlag, New York, 350 p. (2004).

[13] L.D. Landau, E.M. Lifshitz, Statistical Physics, Vol. 5 (Part 1), Butterworth-Heinemann (3rd ed.), 544 p. (1980).

[14] N. Ashcroft, N. Mermin, Solid state physics, Harcourt College Publishers, 826 p. (1976).

[15] A. Brager, A. Schuchowitzky, Journ. Chem. Phys. 14, 569 (1946). doi:10.1063/1.1724202

[16] M. Dupuis, R. Mazo, L. Onzager, Journ. Chem. Phys. 33, 1452 (1960). doi:10.1063/1.1731426

[17] R. Stratton, Journ. Chem. Phys. 37, 2972 (1962). doi:10.1063/1.1733127

[18] A.A. Maradudin, R.F. Wallis, Phys. Rev. 148, 945 (1966). doi:10.1103/PhysRev.148.945

[19] I.A. Viktorov, Sound surface waves in solids, Nauka, Moscow, 287 p. (1981).

[20] F.I. Fedorov, Theory of elastic waves in crystals, Springer, New York, 375 p. (1968). 